\title{
Production of Higgs bosons with large transverse momentum
}

\section{Kirill Kudashkin*}

Karlsruher Institut für Technologie, Institut für Theoretische Teilchenphysik

E-mail: kirill.kudashkin@kit.edu

Recent results on the Higgs plus jet production are reviewed. We have computed the two-loop amplitudes for $g g \rightarrow H g, q \bar{q} \rightarrow H g$ and $q g \rightarrow H q$ at large Higgs transverse momentum. It was the long-missing part of NLO corrections to the Higgs plus jet production above the top mass threshold. This result is combined with the real corrections to produce the Higgs boson transverse momentum distribution at NLO. 


\section{Introduction}

In 2012 a new particle was observed which was later identified as the fundamental scalar boson of the Standard Model (SM). Its properties are in perfect agreement with theoretical predictions within the SM. Now the SM is going through a new period of stress tests where we are increasing the accuracy of our calculations. Both experimental and theoretical physicists are trying to find any signs of discrepancy between Large Hadron Collider (LHC) data and the SM.

The top-Yukawa coupling is a potential source of new findings. The possibility to find new physics comes from the fact that the top-Yukawa coupling $k_{t}$ is known experimentally to a precision of 50\% from the $t \bar{t} H$ production process. It leaves space for a new point-like $g g H$ interaction $k_{g}$. To demonstrate this possibility let us look at the inclusive cross-section for $g g H$ in a scenario where we include a new point-like interaction, that is, $\sigma_{g g \rightarrow H} \sim \alpha_{s}^{2} / v^{2}\left(k_{g}+k_{t}\right)^{2}$ where $k_{g}, k_{t}$ are anomalous couplings. In other words, if we consider the Higgs production inclusively we will be able to constrain only the sum of anomalous couplings [1]. Hence, we must go beyond inclusive searches. Here it should be noted that there was already an attempt to do this experimentally [2].

The Higgs plus jet production is a way to solve this problem. Indeed, if the Higgs is produced with an additional jet, it will result in a complex kinematics which involves both the top quark and the new degree of freedom which is responsible for the new point-like interaction. Assuming the scale of the new physics to be high enough, say $\lambda_{x}=1000 \mathrm{GeV}$, we will have a region $2 m_{\text {top }}<$ $p_{T, H}<\lambda_{x}$ where the top loop is resolved and the new interaction can still be considered pointlike. Increasing $p_{T, H}$-cut will make the contribution of the top loop smaller while the point-like interaction will not change [3]. In this way, we will be able to break the degeneracy between these two anomalous couplings.

In this talk, we addressed the problem that there were no sufficiently accurate calculations for the Higgs plus jet production. Indeed, there was only the LO cross-section available in the high $p_{T, H}$ region for 30 years [4]. To resolve this problem we have calculated NLO corrections in $\alpha_{s}$ to the Higgs boson production at the high $p_{T, H}$ including the top mass effects [5].

\section{NLO corrections to the Higgs plus jet production}

In this section, we consider how the NLO corrections were computed within perturbative QCD. The NLO corrections consist of two parts: real corrections and virtual corrections. The real corrections are a one-loop $2 \rightarrow 3$ process. It has been computed analytically [6]. However, we have used an OpenLoops implementation for convenience [7].

In general, the virtual correction is difficult to compute. It is a four scale problem: $\left\{s, t, m_{H}^{2}, m_{\text {top }}^{2}\right\}$ where $s, t$ are Mandelstam variables, $m_{H}$ is the Higgs mass and $m_{t o p}$ is the mass of the top quark. Depending on transverse momentum $p_{T, H}$ of the Higgs, there is a way to simplify calculations. ${ }^{1}$ In the region where $p_{T, H}^{2} \ll m_{t o p}^{2}$ one gets the following hierarchy of scales: $\left\{s, t, m_{H}^{2}\right\} \ll m_{t o p}^{2}$. When computing master integrals one can employ the large mass expansion based on the observation above.

\footnotetext{
${ }^{1}$ For phenomenological reasons, we are assuming that only the top quark is massive. All other quarks are considered massless.
} 
However, this procedure breaks down if $p_{T, H}^{2} \gg 4 m_{t o p}^{2}$. Indeed, in this region the hierarchy of scales is the following: $m_{H}^{2}<m_{t o p}^{2} \ll\{s, t\}$. In other words, the large mass expansion cannot account for the top loop effects. Thus, one should come up with a different approach. Before reviewing this approach from [8], let us look at the different works.

The most ambitious project is [9], where master integrals are being calculated analytically with the full top mass dependence. While planar master integrals are already calculated, non-planar integrals seem to be more complicated. Indeed, the size of the intermediate results is so large that it gets harder to handle it. Beyond-polylogarithmic functions also complicate the computations. Namely, one will use symbols to handle these large expressions, but there were no symbols for elliptic functions until recently [10]. ${ }^{2}$ More successful way was used in [11]. There, two-loop amplitudes were calculated numerically using pySecDec [12]. It was capable of getting the full top mass dependence. ${ }^{3}$

We advocate a different approach. In general, it is based on differential equations [13]. We will not describe how to get from diagrams to differential equations since there are many papers where it is written in details [8]. Here we would like to note an important feature of the $p_{T, H}^{2} \gg 4 m_{t o p}^{2}$ region. It is readily seen that one can construct two small parameters $m_{H}^{2} / 4 m_{t o p}^{2} \sim 0.13$ and $m_{\text {top }}^{2} / s \ll 1$. Then, it is possible to employ small parameter expansion at the level of differential equations [14]. Namely, the analysis of the differential equations suggests a particular form of the integrals. We came up with the following ansatz

$$
\Im_{i}(\kappa, \eta, z, \varepsilon)=\sum_{j, k, l, m \in Z, n \in N} c_{i, j, k, l, m, n}(z, \varepsilon)\left(m_{\text {top }}^{2} / s\right)^{j-k \varepsilon}\left(m_{H}^{2} / 4 m_{\text {top }}^{2}\right)^{l / 2-m \varepsilon} \log ^{n}\left(m_{H}^{2} / 4 m_{\text {top }}^{2}\right) .
$$

where $\varepsilon$ is the dimension regularization parameter, $z=t / s$ and $c_{i, j, k, l, m, n}(z, \varepsilon)$ are unknown functions which should still be integrated via differential equations. We emphasis that $\{i, j, k, l, m, n\}$ indices has to be truncated at some values, that is, it is a finite series.

Advantages of this method should be noted. First, we are not looking for a canonical form of differential equations. In general, it is not a problem to get a canonical form of differential equations, and at this moment it is better understood than before, but still, it requires some effort. Moreover, some of the differential equations for the two-loop master integrals cannot be put in the canonical form ${ }^{4}$, but our method will work regardless of the differential equation form. Second, we are effectively reducing the four scale problem to the "one" scale problem. Indeed, after inserting the ansatz into differential equations we will have algebraic equations with respect to $\left\{m_{H}^{2} / 4 m_{\text {top }}^{2}, m_{\text {top }}^{2} / s\right\}$ which can be easily solved. At last, the "one" scale problem is well enough understood and can be integrated by known packages [16]. Finishing the integration will lead us to a point where we have to fix integration constants, that is, the boundary conditions.

Computing boundary conditions is the last step in the calculations of the virtual corrections. We emphasize that a specific physical region is considered and our expansion will only work in this region. It will affect what kind of limits we can take. For example, $s \rightarrow 0$ is not possible anymore,

\footnotetext{
${ }^{2}$ see the proceedings of H.Frellesvig

${ }^{3}$ see the proceedings of M. Kerner

${ }^{4}$ Due to elliptic integrals
} 
since $m_{\text {top }}^{2} / s \ll 1$ is not satisfied. In general, we cannot take some convenient limits to compute integration constants. However, it is still possible to apply unitarity cuts [15]. Indeed, the differential equations will have a certain type of singularities which don't coincide with singularities of the corresponding diagram, that is, spurious singularities. Hence, we can set corresponding coefficients to be zero when the integral approaches a singularity. It will result in more algebraic equations and hence allows us to reduce the number of integration constants. It is a very powerful method since we fixed roughly $70 \%$ of all 261 constants with this method. The next way can be described as follows: we note that on amplitude level there are no singularities in the Higgs mass. Indeed, the Higgs always couples to top quarks. Hence, we must constrain how the ansatz should look like. On the differential equation level, we should put to zero all coefficients multiplying any negative powers of the Higgs mass such as $1 / m_{H}$ which also results in additional algebraic equations. We must admit that in few subgraphs it is possible that the external massive line couples to internal massless lines. However, it happened a few times and this case can be treated individually. This is just two methods that we applied to compute integration constants. We used different approaches and it is not possible to review them all here. We refer once again to the author paper [8].

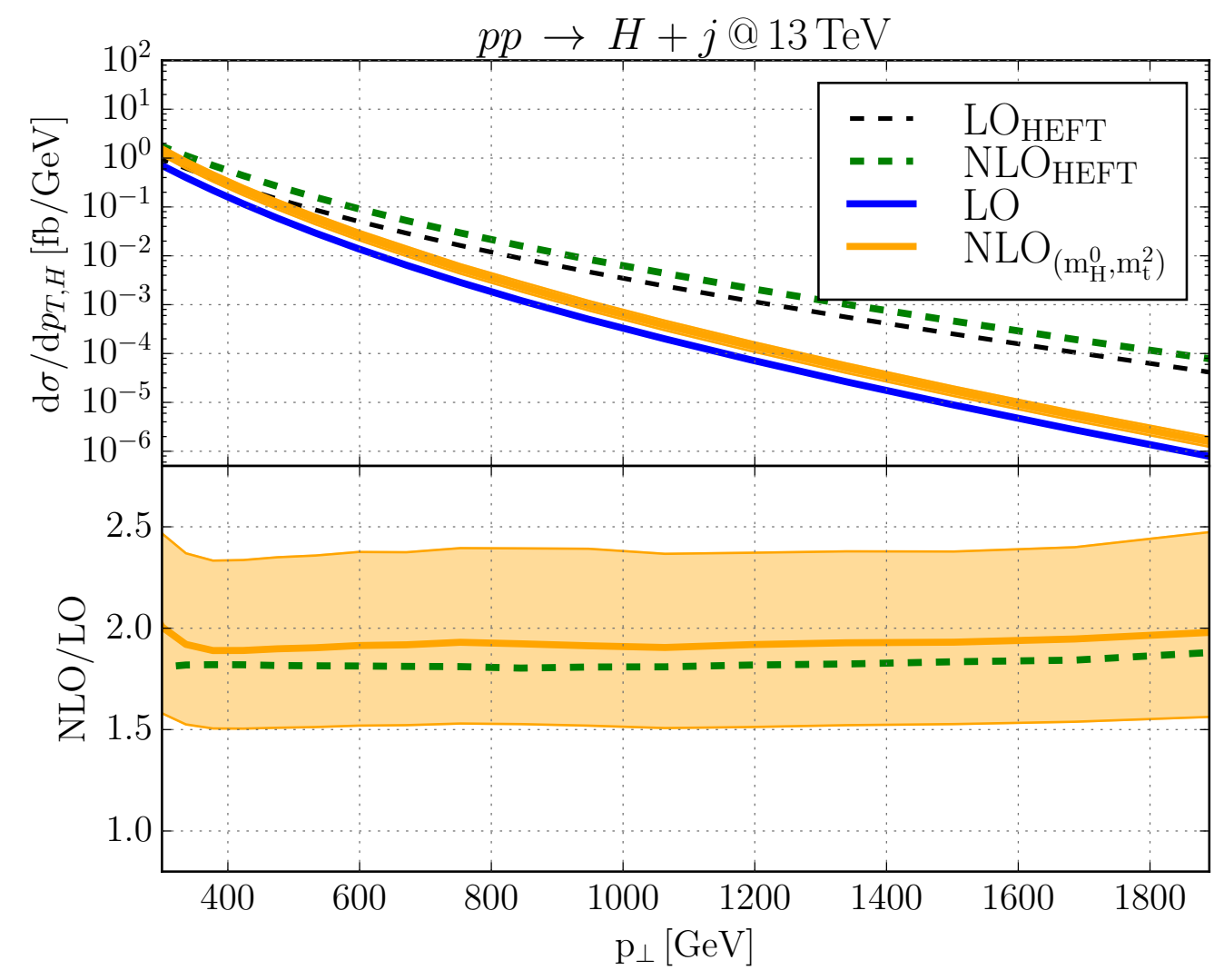

Figure 1: Transverse momentum distribution of the Higgs boson. The upper panel shows absolute predictions at LO and NLO in the small parameter expansion and in the approximation of infinite top mass (HEFT). The lower panel corresponds to the ration between NLO and LO corrections. The bands indicate theoretical uncertainty of the expanded result due to variation of $\mu$. 


\section{Results}

Combining real and virtual corrections allows us to present the Higgs boson transverse momentum distribution. We considered proton collisions at $13 \mathrm{TeV}$. The mass of the Higgs is taken to be $m_{H}=125 \mathrm{GeV}$. The top mass is taken $m_{t o p}=173.2 \mathrm{GeV}$. We used five-flavor scheme and considered bottom-quark to be massless parton in the proton. We used NNPDF 3.0 set of parton distributions with provided $\alpha_{s}$. The renormalization scale is chosen to be

$$
\mu_{0}=H_{T} / 2, H_{T}=\sqrt{m_{H}^{2}+p_{T, H}^{2}}+\sum_{j} p_{T, j}
$$

We estimate theoretical uncertainties by varying the renormalization and the factorization scales $\mu$ by a factor of two with respect to the central value. The final results are shown in Fig. 1 .

We note that the ratio between NLO and LO correction ${ }^{5}$ is flat and equal $\sim 1.9$ for $p_{T, H}>$ $400 \mathrm{GeV}$. The top mass effects estimated by comparing $K$-factors for the expanded case and HEFT. At $p_{T, H}=400 \mathrm{GeV}$ this ratio is equal $K_{S M} / K_{H E F T}=1.04$ and at $p_{T, H}=1000 \mathrm{GeV}$ this ratio is equal $K_{S M} / K_{H E F T}=1.06$. The theoretical uncertainty is reduced from $40 \%$ at LO to $20 \%$ at NLO.

\section{Summary and outlook}

Master integrals were computed for virtual corrections to the Higgs plus jet production in the large $p_{T, H}$ region. The virtual corrections are then combined with the existing real corrections in order to produce the Higgs boson transverse momentum distribution. Theoretical uncertainties are estimated to have value $20 \%$. The main result is presented in Fig. 1. The method of small parameter expansion has proved itself to be useful in this example. It can be applied to different LHC-related processes where the similar hierarchy of scales is present. Our result can be used to constrain anomalous couplings in some BSM scenarios which modify the Higgs sector of SM.

\section{Acknowledgements}

K.K thanks Kirill Melnikov, Christopher Wever, Jonas M. Lindert and Hjalte Frellesvig for useful discussions and for preparations to this talk at the Loops\&Legs 2018. The research of K.K. is supported by the DFG-funded Doctoral School KSETA (Karlsruhe School of Elementary Particle and Astroparticle Physics).

\section{References}

[1] C. Grojean, E. Salvioni, M. Schlaffer and A. Weiler, JHEP 1405, 022 (2014) doi:10.1007/JHEP05(2014)022 [arXiv:1312.3317 [hep-ph]].

[2] A. M. Sirunyan et al. [CMS Collaboration], Phys. Rev. Lett. 120 (2018) no.7, 071802 doi:10.1103/PhysRevLett.120.071802 [arXiv:1709.05543 [hep-ex]].

[3] A. Banfi, A. Martin and V. Sanz, JHEP 1408, 053 (2014) doi:10.1007/JHEP08(2014)053 [arXiv:1308.4771 [hep-ph]].

\footnotetext{
${ }^{5} K=\sigma_{N L O} / \sigma_{L O}$ factor
} 
[4] R. K. Ellis, I. Hinchliffe, M. Soldate and J. J. van der Bij, Nucl. Phys. B 297 (1988) 221. doi:10.1016/0550-3213(88)90019-3

[5] J. M. Lindert, K. Kudashkin, K. Melnikov and C. Wever, Phys. Lett. B 782 (2018) 210 doi:10.1016/j.physletb.2018.05.009 [arXiv:1801.08226 [hep-ph]].

[6] V. Del Duca, W. Kilgore, C. Oleari, C. Schmidt and D. Zeppenfeld, Phys. Rev. Lett. 87 (2001) 122001 doi:10.1103/PhysRevLett.87.122001 [hep-ph/0105129].

[7] F. Cascioli, P. Maierhofer and S. Pozzorini, Phys. Rev. Lett. 108 (2012) 111601 doi:10.1103/PhysRevLett.108.111601 [arXiv:1111.5206 [hep-ph]].

[8] K. Kudashkin, K. Melnikov and C. Wever, JHEP 1802 (2018) 135 doi:10.1007/JHEP02(2018)135 [arXiv:1712.06549 [hep-ph]].

[9] R. Bonciani, V. Del Duca, H. Frellesvig, J. M. Henn, F. Moriello and V. A. Smirnov, JHEP 1612 (2016) 096 doi:10.1007/JHEP12(2016)096 [arXiv:1609.06685 [hep-ph]].

[10] J. Broedel, C. Duhr, F. Dulat and L. Tancredi, JHEP 1805 (2018) 093 doi:10.1007/JHEP05(2018)093 [arXiv:1712.07089 [hep-th]].

[11] S. P. Jones, M. Kerner and G. Luisoni, Phys. Rev. Lett. 120 (2018) no.16, 162001 doi:10.1103/PhysRevLett.120.162001 [arXiv:1802.00349 [hep-ph]].

[12] S. Borowka, G. Heinrich, S. Jahn, S. P. Jones, M. Kerner, J. Schlenk and T. Zirke, Comput. Phys. Commun. 222 (2018) 313 doi:10.1016/j.cpc.2017.09.015 [arXiv:1703.09692 [hep-ph]].

[13] A. V. Kotikov, Phys. Lett. B 254 (1991) 158. doi:10.1016/0370-2693(91)90413-K

[14] K. Melnikov, L. Tancredi and C. Wever, JHEP 1611 (2016) 104 doi:10.1007/JHEP11(2016)104 [arXiv:1610.03747 [hep-ph]].

[15] R. E. Cutkosky, J. Math. Phys. 1 (1960) 429. doi:10.1063/1.1703676

[16] O. Gituliar and V. Magerya, Comput. Phys. Commun. 219 (2017) 329 doi:10.1016/j.cpc.2017.05.004 [arXiv:1701.04269 [hep-ph]]. 